Linguistique, littérature, didactique

$177-178$ | 2018

Langage oral à l'école maternelle. Étude d'un corpus

homogène

\title{
Effets possibles de la mémorisation partielle d'un texte écrit sur les productions orales d'élèves de maternelle
}

Potential effects of partial memorization of a written text upon kindergarten pupil's oral productions

\section{Marceline Laparra}

\section{OpenEdition}

Journals

Édition électronique

URL : http://journals.openedition.org/pratiques/4392

DOI : 10.4000 /pratiques.4392

ISSN : 2425-2042

Éditeur

Centre de recherche sur les médiations (CREM)

Référence électronique

Marceline Laparra, «Effets possibles de la mémorisation partielle d'un texte écrit sur les productions orales d'élèves de maternelle », Pratiques [En ligne], 177-178| 2018, mis en ligne le 20 juillet 2018, consulté le 30 avril 2019. URL : http://journals.openedition.org/pratiques/4392 ; DOI : 10.4000/ pratiques. 4392

Ce document a été généré automatiquement le 30 avril 2019.

(c) Tous droits réservés 


\title{
Effets possibles de la mémorisation partielle d'un texte écrit sur les productions orales d'élèves de maternelle
}

\author{
Potential effects of partial memorization of a written text upon kindergarten \\ pupil's oral productions
}

Marceline Laparra

1 Plusieurs points de vue peuvent être adoptés pour travailler sur un corpus d'oral scolaire. Celui qui nous intéresse est constitué par la transcription d'une même séance réalisée dans douze classes de maternelle avec un groupe d'une dizaine de «Grands » qui devaient se passer la parole selon un protocole rigoureux (Péroz, 2010) pour raconter collectivement une histoire qui leur avait été lue préalablement et pour dire ensuite ce qu'ils pensaient des différents personnages. Un corpus de ce type permet par exemple d'examiner les interactions entre les différents acteurs ou d'observer la manière de raconter des élèves pour interroger leur degré de maitrise du discours narratif. Il permet également de se demander s'il y a des procédures d'étayage d'une prise de parole à une autre.

2 Mais dans tous ces cas de figure, on laisse dans l'ombre deux paramètres essentiels pour l'étude des apprentissages scolaires. En procédant ainsi on se focalise sur ce qui se passe à l'oral et on laisse dans l'ombre le texte écrit qui a été lu aux élèves avant le début de la séance. Or les élèves ne passent pas «mécaniquement » du monde de l'écrit à celui de l'oral et inversement à la fin de l'école maternelle. Ils sont plutôt confrontés à un univers hybride où oral et écrit et plus largement oralité et littératie s'interpénètrent en permanence (Laparra \& Margolinas, 2016). Et surtout on néglige la manière dont les élèves investissent la situation installée par chaque enseignant, alors même que celle-ci conditionne les productions des élèves. 
Dans le corpus proposé, différents phénomènes se combinent autour de la langue du texte source et de la situation didactique et peuvent expliquer certaines des différences que l'on observe d'un élève à un autre : d'une part, la disponibilité du texte source varie d'un élève à un autre et d'un moment de la séance à un autre. Cette variation place les élèves dans des situations qui se modifient pour un même élève ou d'un élève à un autre. D'autre part, la proximité existant entre la langue écrite du texte source et l'oral des élèves est plus ou moins grande et agit sur ce degré de disponibilité.

Avant toute étude des productions des élèves s'impose donc une analyse minutieuse de la situation réellement investie par les élèves et une description de la langue du texte source.

\section{Spécificités de la situation des différentes séances du corpus}

\section{Une situation d'engendrement de l'histoire très particulière}

Dans la première partie de chaque séance la manière dont les élèves investissent la situation didactique qui leur est proposée (Brousseau, 1998) dépend de plusieurs paramètres :

1. Le texte source a été lu antérieurement à la séance une ou plusieurs fois sans que jamais soient montrées aux élèves les illustrations de l'album (pour les différents modes de restitution d'une histoire en Maternelle cf. F. Grossmann [2000] et S. Bonnéry [2014]). L'accès à l'univers de référence ne peut donc être que linguistique. Une telle démarche est loin d'être majoritaire en maternelle (Péroz, 2010) mais les élèves des classes du corpus y sont habitués.

2. Les élèves ont le droit de redire ce qui a déjà été dit par leurs camarades.

3. Ils n'ont pas, sauf dans deux classes et encore de manière fugitive au début de la séance, à respecter l'ordre chronologique de l'histoire. Il ne leur est pas demandé de «raconter l'histoire » mais de dire ce dont ils se souviennent. Ils peuvent donc, à condition de suivre les règles de prise de parole instaurées dans le groupe, dire chacun à leur tour ce qui leur revient en mémoire. Peu importe alors que ce qu'ils évoquent se situe au début, au milieu ou à la fin de l'histoire.

4. Ils doivent se soumettre à une règle d'exhaustivité. Dans plusieurs classes les enseignants relancent les élèves en leur demandant avec insistance s'ils ont tout dit.

Tout ceci a un effet sur la tâche que les élèves ont à réaliser. Ils doivent moins raconter une histoire qu'en rappeler à tour de rôle les éléments dont ils se souviennent. L'histoire est en quelque sorte décomposable en « morceaux » détachables les uns des autres et leur tâche est de les retrouver les uns après les autres. Comme ils n'ont pas à respecter un ordre quelconque d'engendrement, comme l'ordre chronologique, ils sont dépourvus de repères pour en faire une énumération réussie (Briand, 1993). Ils ne peuvent donc qu'omettre certains de ces "morceaux » et au contraire en proposer d'autres plusieurs fois et ceci d'autant plus facilement qu'ils sont autorisés à redire ce qui a déjà été dit par d'autres.

7 Or, d'une part, d'une classe à l'autre l'oralisation du texte Danger dans le potager ne s'est pas toujours passée de manière identique : il a été lu une fois ou plusieurs, de manière segmentée ou non, le jour de la séance ou un ou plusieurs jours avant. D'autre part, les 
élèves ont pu entendre oraliser cette histoire antérieurement soit en moyenne section soit à la maison, l'album ayant un grand succès auprès des jeunes publics et des adultes qui les ont en charge. La familiarisation avec le texte est donc variable au début de la séance d'une classe à une autre et d'un élève à un autre.

Dans la première partie de la séance, les élèves qui interviennent, en essayant de respecter les différentes contraintes qui sont les leurs, «apportent " chacun à leur tour un petit morceau de l'histoire, mais certains d'entre eux le font alors en restituant des petits « morceaux » du texte source, ce qui contribue à distinguer leurs productions de celles de leurs camarades. Dans la deuxième partie de la séance, une modification de la situation intervient qui explique que le texte source s'efface presque complétement pour ces élèves : ils doivent désormais comme leurs camarades dire ce qu'ils pensent des personnages principaux. Néanmoins leurs interventions se différencient toujours fortement des autres.

9 Mais si le texte source peut contribuer à apporter aux élèves certaines des connaissances mises en jeu par eux dans la gestion de la situation, c'est peut-être aussi parce qu'il est rédigé dans une langue qui tout en respectant tous les codes de la langue écrite conserve certaines des caractéristiques de l'oral auquel sont habitués les élèves (François, 1974; Laparra, 1982; Gadet, 1989; Blanche-Benveniste, 1997), à savoir un oral produit dans l'immédiateté et en coprésence, ce qui permet d'avoir une référence directe pour certains objets du monde.

\section{Écart au plan syntaxique entre le texte support et la langue parlée utilisée en classe}

10 Avant l'étude de la syntaxe de ce texte, quelques remarques s'imposent sur sa structure narrative et son lexique. Danger dans le potager présente certaines difficultés pour des élèves âgés de cinq ans, quel que soit leur degré de familiarité avec ce type d'univers littératié.

11 Certes la structure de l'histoire est relativement simple. La narration à thème constant développe les différentes péripéties d'une course-poursuite entre deux acteurs aux rôles bien définis. Mais celles-ci sont nombreuses et pour certaines déconcertantes. De manière peu explicite sont confrontés deux espaces qui présentent des caractéristiques opposées à ce que seraient les leurs dans le monde que fréquentent les élèves : l'espace ouvert et non domestiqué est celui qui est sûr et l'espace clos et domestiqué est celui qui est dangereux. Cela ne va pas de soi pour de jeunes enfants qui commencent juste à découvrir des mondes inversés où des animaux sauvages sont anthropomorphisés sous les traits de petits humains poursuivant leur apprentissage de la civilisation.

12 Néanmoins les interventions des élèves dans les différentes classes ne manifestent aucune difficulté de compréhension : on n'observe aucune confusion de personnages et ceux-ci se voient attribuer les propos et les rôles qui sont les leurs. Seul l'épisode de la chatte blanche leur pose des problèmes; mais ce n'est guère étonnant, son insertion dans l'histoire pouvant être énigmatique, même pour des lecteurs plus experts.

13 Le lexique du texte ne présente que peu de termes que l'on peut supposer absents du vocabulaire passif d'élèves de cinq ans: civet, potager, remise pour les termes concrets et intrépide, perturbé et émotions pour les termes abstraits ont été expliqués par les enseignants lors de la lecture du texte et sont souvent repris dans les interactions à bon 
escient. Donc les difficultés lexicales et narratives que contient ce texte sont assez facilement surmontées par les élèves qui interviennent dans la classe.

Il en va pratiquement de même au plan syntaxique. Le texte s'il s'écarte relativement souvent de la syntaxe de la langue parlée en reste cependant proche. La très grande majorité des énoncés présente une organisation qui est celle du français oral : un pronom personnel animé singulier (ou un syntagme nominal animé singulier dépourvu d'expansion) suivi immédiatement d'un verbe à haute fréquence d'emploi, lui-même suivi d'un ou deux syntagmes nominaux compléments qui eux peuvent être inanimés, pluriels et expansés par un adjectif, un autre syntagme nominal ou une relative introduite par qui ou que (Dubois, 1965). On ne trouve dans le texte que trois groupes nominaux inanimés sujets («la course reprend», «le bouton du gilet se prend dans le filet», "sa queue frétille »). Peu d'éléments sont antéposés au sujet (« sans réfléchir », « comme le lui a dit sa cousine $»)$.

15 Mais dire cela ne veut pas dire que le texte cherche à imiter la langue orale. La structure syntaxique est bien celle de l'écrit : il n'y a pas de sujet présentant une dislocation du type Pierre il..., les négations sont doubles et beaucoup de syntagmes nominaux postposés au verbe ont des expansions comportant des combinaisons de plusieurs adjectifs ou des combinaisons d'adjectifs et de syntagmes nominaux ou de relatives dont certaines peuvent être enchâssées et être introduites par un relatif autre que qui et que (les racines d'un vieux pin centenaire, le potager interdit de monsieur Grégory, l'endroit par lequel il est entré, un poisson rouge dont elle..., le filet qui recouvre le plant de groseilles qu'il est en train...). Ces difficultés sont particulièrement denses dans un court paragraphe qui présente la dernière péripétie de la poursuite, péripétie qui, nous l'avons vu, est en outre très obscure. S'y trouve l'énoncé le plus difficile du texte (ne sachant pas si, comme le lui a dit son cousin, les chats sont...) puisqu'il comporte une inversion d'un sujet et l'insertion d'une proposition avant le sujet de la complétive. Tout concourt à ce que les élèves n'arrivent pas à raconter ce passage. Mais il est le seul dans ce cas. Les autres paragraphes confrontent les élèves à une langue écrite se coulant souvent dans le moule syntaxique de la langue parlée.

16 La relative proximité entre la langue écrite du texte et la langue parlée permet que certaines prises de parole des élèves se démarquent quelque peu de l'oral le plus spontané et cela d'autant plus que ce n'est pas la logique des interactions qui guide la sélection thématique des propos (Laparra, 1982) mais une contrainte de la situation didactique et que la convocation de l'univers référentiel ne se fait pas dans la connivence née de la coprésence des locuteurs mais par l'existence dans la mémoire des intervenants d'un univers référentiel de nature textuel.

17 L'oral à l'école est soumis à de nombreuses variations : cela va de l'oral conversationnel entre pairs, lorsque par exemple les élèves conversent entre eux tout en procédant à une tâche, jusqu'à un oral très proche d'un écrit oralisé quand par exemple le maitre résume pour la classe ce qu'il convient de retenir d'une activité. L'oral scolaire est instable, se modifiant sans cesse au gré des changements qui affectent les situations investies par les différents partenaires de la classe (Laparra, 2003). Quand nous parlons ici de la syntaxe du français parlé nous renvoyons aux modes d'organisation syntaxique les plus souvent observés dans les échanges verbaux produits dans l'immédiateté et en coprésence et dont la fréquence explique qu'ils soient très rapidement acquis par les jeunes enfants (François, 1974). 


\section{Analyse des productions des élèves}

\section{Comparaison des productions des élèves qui restituent des « morceaux du texte source » et de celles des élèves qui ne le font pas dans la première partie de la séance}

Nous avons vu en analysant la situation didactique du corpus qu'un petit groupe d'élèves se distinguent des autres dans plusieurs classes. Il s'agit de ceux qui semblent avoir été mieux que d'autres capables de mémoriser presque littéralement des «morceaux » du texte source et de les faire leurs dans leurs réponses. Dans la séance 1: Mourad et Shona et à un moindre degré Sohaib; dans la séance $3:$ Malo; dans la séance $6:$ Marco, Leslie et Emilie; dans la séance 8 : Viviane, Sergueï et Tania; dans la séance 11 : Akem, Julianne, Lise et Théophile. Si leurs camarades racontent des morceaux de l'histoire en oral spontané, eux peuvent aussi par moments restituer des morceaux du texte source. Mais comme ils sont dans une situation d'interaction verbale, ces reprises de segments du texte sont insérées dans un discours oral. Les énoncés produits présentent alors à la fois des caractéristiques de la langue écrite et de la langue orale. Comparons ce que disent Oudriss et Shona (séance 1), les reprises littérales figurent en italique.

Tableau 1. Un exemple d'insertion du texte source

\begin{tabular}{|l|l|l|l|}
\hline Séance & $\mathbf{N}^{\mathbf{0}}$ & Loc & Interventions \\
\hline 1 & 5 & Oudriss & $\begin{array}{l}\text { après Pierre/ après il a dit/ après il a fait atchoum/ et après il a fait et } \\
\text { après/ et après Pierre et après Pierre il a été dans le potager et la maman } \\
\text { et la maman elle a dit ne passez pas dans le potager }\end{array}$ \\
\hline 1 & 10 & Shona & $\begin{array}{l}\text { eh bien quand monsieur Grégory il est arrivé/ il a dit/ Pierre/ qu'est-ce } \\
\text { qu'il y a dans ce potager/ il a vu Pierre/ et monsieur Grégory il a dit je te } \\
\text { transformerai en pâté/ après Pierre il a couru/ et monsieur Grégory il l'a } \\
\text { les pommes de terre et il s'est accroché dans un légume et après au moment } \\
\text { où il fallait il enlève son gilet/ monsieur Grégory s'est retourné/ il a entendu } \\
\text { atchoum derrière lui/ il passe entre les jambes de monsieur Grégory et après } \\
\text { Pierre il rentre dans la remise }\end{array}$ \\
\hline
\end{tabular}

Oudriss tente de narrer certains éléments de l'histoire dans le désordre. Il hésite très souvent; certains énoncés sont inachevés. Le seul connecteur est et après, les verbes sont dire, faire, aller, passer; plusieurs sujets nominaux sont extraposés avec reprise pronominale. Même si son intervention est relativement longue (48 mots) et pourrait le ranger parmi les " moyens parleurs ", elle est difficile à suivre et donne l'impression à qui l'écoute que son auteur n'est capable que de dire des bribes d'une histoire très maladroitement dans une langue conversationnelle.

Shona elle aussi utilise des pronoms sujets avec extraposition du nom; ses phrases sont coordonnées par et et et après; les verbes sont à haute fréquence, voire familiers comme dans « il l'a coursé ». Mais elle insère dans sa narration à la fois des reformulations très 
proches du texte source et des reprises à l'identique ; elle reformule : «juste à temps » en «au moment où il fallait» et "le bouton de son gilet se prend dans le filet » en «il s'est accroché dans un légume ». Et elle introduit de légères variantes dans les reprises qu'elle fait du texte : «il passe entre les jambes »/« il passe sous les jambes » et «il enlève son gilet »/«ierre vient d'enlever son gilet ». Mais surtout elle est capable de restituer à l'identique une phrase construite à l'aide du couple « l'un... l'autre ». L'ensemble donne l'impression d'une narration fluide et aisée. L'appui sur le texte source permet à Shona de s'essayer à produire des patrons d'énoncés différant quelque peu de ceux dont on use habituellement dans la langue parlée.

Il est à remarquer que l'écart existant entre les interventions d'élèves comme Oudriss et celles d'élèves comme Shona est peut-être légèrement accentué par la manière dont ont été réalisées les transcriptions. Celles-ci ont été faites pour chaque séance par l'enseignant de la classe qui n'avait pu être formé qu'imparfaitement au respect des exigences d'une transcription savante. En témoigne le fait que les négations doubles y ont une fréquence anormalement élevée et que les ratés du discours y ont à l'inverse une faible densité. Or ceux qui comme nous ont vu faire leurs étudiants savent que des débutants en la matière ont tendance à moins noter certains traits caractéristiques de la langue parlée comme les hésitations ou les répétitions quand le locuteur est supposé habile et au contraire à les relever plus systématiquement pour les locuteurs supposés peu compétents.

Les élèves du type de Shona se montrent capables d'apporter au segment du texte qu'ils reprennent les modifications nécessitées par la cohérence de leur propos. Ils procèdent à des ajustements dans les chaines de désignateurs, ou changent le régime temporel du texte source en passant du présent dit de narration à une alternance juste de l'imparfait et du passé composé. Ils "conversationnalisent» en quelque sorte le texte écrit. Deux élèves en sont un bon exemple, Akem et Théophile.

Dans la première partie de la séance 11 , ceux-ci font des interventions qui sont presque entièrement constituées d'un assemblage de plusieurs segments du texte repris presque mot à mot avec juste les ajustements décrits ci-dessus.

Tableau 2. Un exemple de reprise presque littérale

\begin{tabular}{|l|l|l|l|}
\hline Séance & $\mathbf{N}^{\mathbf{0}}$ & Loc & Intervention \\
\hline 11 & 2 & Akem & $\begin{array}{l}\text { il a dit la course folle reprend/ la course folle reprend de plus belle [paragraphe } 9^{1} \\
\text { /ans l'arrosoir mais quelle erreur!/ l'arrosoir était plein d'une eau glacée/ il ne } \\
\text { bouge pas parce que monsieur Grégory s'approche/ et d'un coup a::a:::atchoum/ } \\
\text { monsieur Grégory se retourne [paragraphe 8]et la course folle se reprend de plus } \\
\text { belle [paragraphe 9]/ }\end{array}$ \\
\hline
\end{tabular}

Akem ne garde le présent que pour deux verbes; il remplace quand il le faut le pronom il par le prénom Pierre; il ajoute un connecteur mais; il modifie sous en entre, approche en s'approche, tout à coup en d'un coup, reprend en se reprend; il omet les termes juste et à moitié ; il réunit des segments de trois paragraphes différents, pas toujours dans l'ordre. Et au prix de toutes ces opérations effectuées au fur et à mesure de sa production il donne l'impression à celui qui l'écoute d'être bien celui qui en est l'auteur. 
Tableau 3. Production où alternent citation du texte source et reformulation

\begin{tabular}{|l|l|l|l|}
\hline Séance & $\mathbf{N}^{\mathbf{0}}$ & Loc & Intervention \\
\hline 11 & 11 & Théophile & $\begin{array}{l}\text { monsieur monsieur Grégory// Pierre il a mangé il a mangé de la salade } \\
\text { deux ou trois gros haricots verts et d'énormes radis [paragraphe 5]// tout à } \\
\text { coun bruit l'effraie/ euh un bruit inquiétant/ que se passe-t-il dans ce } \\
\text { potager si tranquille// c'est monsieur Grégory qui vient de l'apercevoir alors } \\
\text { qu'il était en train de planter des jeunes choux [paragraphe 6] }\end{array}$ \\
\hline
\end{tabular}

Théophile ne reproduit pas complètement la description du festin fait par Pierre dans le potager qui se trouve dans le paragraphe 5 du texte: il commence par reformuler l'énoncé il goûte quelques belles laitues bien croquantes en il a mangé de la salade mais il en restitue ensuite mot à mot la fin. Puis son intervention est une reprise presque littérale du début du paragraphe 6, moins le premier adjectif du syntagme un bruit étrange et inquiétant et la subordonnée de temps par laquelle commençait ce paragraphe alors qu'il termine tranquillement un concombre. Théophile d'une certaine manière raconte d'abord avec ses propres mots comment Pierre a été interrompu au milieu de son repas et une fois le début de son intervention assuré, il continue en reprenant deux longs segments du texte mis bout à bout; il change seulement le régime temporel. Trois autres interventions de Théophile seront presque uniquement des reprises fidèles du texte source (S11-30; S11-46; S11-51).

Tous ces élèves rappelons-le ne savent pas lire et n'ont eu accès au texte qu'au travers de l'oralisation qui leur en a été faite. Pourtant à des degrés divers ils se montrent capables dans une situation d'interaction verbale de produire des énoncés dont ils empruntent beaucoup d'éléments au texte écrit mais qu'ils donnent l'impression de faire leurs, ce dont témoigne la cohérence des modifications qu'ils y apportent, au point que le récepteur peut les créditer de la capacité d'enrichir la syntaxe de la langue parlée en produisant par exemple des sujets inanimés ou des expansions variées de syntagmes nominaux.

\section{Comparaison des productions des élèves qui restituent des « morceaux » du texte source dans la deuxième partie de la séance avec celles de la première partie}

27 Les règles données par les maitres pour la deuxième partie de la séance modifient la situation dans laquelle les élèves se trouvent. Ils doivent dire ce qu'ils pensent de la conduite des personnages principaux. Beaucoup vont alors le faire en s'appuyant sur leur expérience personnelle (par exemple dans la séance 8, Viviane en S8-88 ou S8-124). Les retours au texte source se font plus rares et les interventions des élèves qui s'étaient appuyés fortement sur lui relèvent beaucoup plus de la langue parlée spontanément.

Tableau 4. Exemple de productions langagières spontanées

\begin{tabular}{|l|l|l|l|}
\hline Séance & $\mathrm{N}^{\mathrm{o}}$ & Loc & Interventions \\
\hline
\end{tabular}




\begin{tabular}{|l|l|l|l|}
\hline 11 & 61 & Akem & $\begin{array}{l}\text { il est gentil mais il a pas été très sage/ il a pas écouté sa maman/ et et et et } \\
\text { et il a arrivé à sortir du potager et à aller chez son terrier }\end{array}$ \\
\hline 11 & 91 & Akem & $\begin{array}{l}\text { on était gentil/ on était sage/ on a écouté notre maman/ on est allé dire/ la } \\
\text { maman nous a dit qu'on n'a pas/ aller quelque part on le fait pas// }\end{array}$ \\
\hline
\end{tabular}

Akem produit ici des phrases réduites au schéma pronom-verbe (être, aller, dire, arriver, faire, sortir, écouter) -adjectif attribut ou complément sans expansion. Il hésite et coordonne ses propositions par et.

Tableau 5. Inachèvement d'énoncés

\begin{tabular}{|l|l|l|l|}
\hline Séance & $\mathbf{N}^{\mathbf{0}}$ & Loc & Interventions \\
\hline 11 & 99 & Théophile & $\begin{array}{l}\text { c'est pas trop bien de fermer tout à clé quand même parce que si nous } \\
\text { nous/ les petits lapins i(ls) pourront pas entrer et la mère aussi elle } \\
\text { pourra pas entrer// }\end{array}$ \\
\hline 11 & 102 & Théophile & $\begin{array}{l}\text { à chaque fois que/ je serais monté à chaque fois pour regarder si } \\
\text { lapereaux/ j'aurais laissé tout ouvert je serais resté// j'aurais regardé } \\
\text { personne pouvait entrer parce qu'on sait jamais si i(l) a des personnes } \\
\text { qui sont encore plus petites que le trou// }\end{array}$ \\
\hline 11 & 110 & Théophile & $\begin{array}{l}\text { euh les lapins ça sait comment on fait un potager/ non les lapins ça } \\
\text { sait pas comment on fait un potager// }\end{array}$ \\
\hline
\end{tabular}

Théophile laisse plusieurs énoncés inachevés; ceux-ci sont souvent réduits dans ces trois répliques au pronom sujet et au verbe; ils sont simplement juxtaposés et comportent parfois des extrapositions du sujet quand il est nominal.

30 Même si dans cette deuxième partie les interventions de ces élèves sont plus longues que celles de beaucoup de leurs camarades, elles présentent un écart très important avec celles qui avaient été les leurs dans la première partie de la séance. Ceci montre que l'effet dû à la présence en arrière-plan d'un texte écrit dont la langue est à la fois proche et éloignée de la leur existe tant que le recours à ce dernier est postulé par la situation. Il s'efface dès que ce n'est plus le cas, ce qui se passe quand disparait l'exigence de dire tout ce dont on se souvient. Le bénéfice qui en a résulté au plan linguistique pour ceux qui ont alors réussi à faire leurs des segments du texte, même s'il est remarquable, semble donc fugitif.

\section{Effets de la mémorisation d'éléments du texte source par certains élèves sur leur capacité interprétative}

31 Néanmoins à la fois dans la première partie de la séance et dans la deuxième partie, ces élèves paraissent capables, plus que ceux de leurs camarades qui ne restituent jamais aucun « morceau du texte", de prendre une distance (Bautier, 1995) avec la seule tâche de verbalisation de tous les épisodes de la course-poursuite. Tout semble se passer pour 
eux comme si l'appui sur le texte source pour restituer des "morceaux» de l'histoire produisait un allégement de leur tâche cognitive: ils n'ont pas alors à se soucier de mettre en mots l'univers de référence, ce qui pèse sur les autres élèves et sont de ce fait plus libres de se poser des questions sur des points importants de l'histoire. Ils peuvent chercher à en expliciter certains points, à mettre en relation ou à caractériser certains faits ou comportements des personnages. Par exemple Akem explique pourquoi la maman donne de la tisane à Pierre. Le texte disait seulement : «Sa maman [...] lui donne une tisane de camomille. Je ne sais pas ce que tu as, mais avec ça tu passeras une bonne nuit ». Akem dit l'effet qu'aura la camomille.

Tableau 6. Un exemple de justification

\begin{tabular}{|l|l|l|l|}
\hline Séance & $\mathbf{N}^{\mathbf{0}}$ & Loc & Intervention \\
\hline 11 & 55 & Akem & $\begin{array}{l}\text { il il il saute de son fenêtre/ il il rentre dans la maison/ il se met dans son lit } \\
\text { et après sa maman lui donne une tisane de camomille comme ça les trucs } \\
\text { qu'il a passés de la journée lui reviennent plus }\end{array}$ \\
\hline
\end{tabular}

Akem utilise un lexique familier pour résumer les évènements que Pierre a vécus, les trucs qu'il a passés de la journée, mais il arrive néanmoins à se représenter que ces évènements ont été perturbants et qu'il est donc nécessaire de les oublier.

Le texte ne dit pas que le potager est un lieu clos où il est difficile d'entrer et d'où il est tout aussi difficile de sortir ; il le fait seulement indirectement, puisque Pierre « se faufile sous la barrière ». Il ne raconte pas comment Pierre en est sorti; il dit juste que Pierre cherche désespérément à sortir du potager » et qu'il «ne s'arrête de courir qu'une fois arrivé dans la douceur de son terrier ». Plusieurs élèves vont parler de cette sortie.

Tableau 7. Une formulation de l'épisode présentant de nombreux implicites

\begin{tabular}{|l|l|l|l|}
\hline Séance & $\mathbf{N}^{\mathbf{o}}$ & Loc & Intervention \\
\hline 11 & 61 & Akem & $\begin{array}{l}\text { il est gentil mais il a pas été très sage/ il a pas écouté sa maman/ et et et et } \\
\text { et il a arrivé à sortir du potager et à aller chez son terrier }\end{array}$ \\
\hline
\end{tabular}

Akem en une seule intervention rapproche la situation initiale et la situation finale. Marco lui à la barrière du début oppose un élément matériel qu'il imagine.

Tableau 8. Une reformulation de l'épisode de l'arrosoir

\begin{tabular}{|l|l|l|l|}
\hline Séance & $\mathbf{N}^{\mathbf{0}}$ & Loc & Intervention \\
\hline 6 & 20 & Marco & $\begin{array}{l}\text { at après il va dans l'arrosoir et l'eau il était tellement tellement gelée qu'il } \\
\text { s'approche de l'arrosoir/ et après il reprend cette course folle/ et après il a } \\
\text { trouvé la porte }\end{array}$ \\
\hline
\end{tabular}


Marco reformule avec ses mots l'épisode de l'arrosoir en donnant les mêmes informations que le texte (pas plus que ce dernier il ne dit comment Pierre sort de l'arrosoir); mais pour lui, si la course folle de Pierre se termine bien, c'est qu'il a réussi à trouver la sortie qu'il cherchait, à savoir la porte du potager. De la même manière le texte évoque comment le gilet de Pierre se prend dans un filet; mais il ne dit pas que de ce fait Pierre est prisonnier. Puis il rapporte que Pierre enlève à temps son gilet mais pas que c'est cette action qui lui permet de se sortir du piège dans lequel il était pris. Rappelons que construire les relations de causalité existant entre ces actes successifs ne va pas de soi pour de jeunes élèves. Certains y arrivent cependant, comme Émilie.

Tableau 9. Une verbalisation réussie de la suite d'actions

\begin{tabular}{|l|l|l|l|}
\hline Séance & $\mathbf{N}^{\mathbf{0}}$ & Loc & Intervention \\
\hline 6 & 17 & Emilie & $\begin{array}{l}\text { après il a trouvé une solution/ il enlève sa veste et il va dans la remise pour } \\
\text { aller dans l'arrosoir et il a fait une erreur parce que l'eau elle était glacée// }\end{array}$ \\
\hline
\end{tabular}

Dans cette intervention Emilie fait à deux reprises un lien entre deux actions; dans le premier cas elle se trompe (pour aller dans l'arrosoir) dans le deuxième elle a raison (parce que l'eau était glacée) ; mais ce qui est intéressant c'est qu'elle soit capable de mettre en relation deux faits qui ne l'étaient pas explicitement dans le texte source. D'autres élèves caractérisent un évènement ou une situation. Par exemple le texte s'ouvre sur l'interdiction qui est faite aux petits lapins d'aller dans le potager de Monsieur Grégory et sur la justification qu'en donne la maman. Sergueï réussit à expliquer que seul des lapins Pierre conteste cette justification, ce qui peut expliquer sa désobéissance.

Tableau 10. La contestation de Pierre le Lapin

\begin{tabular}{|l|l|l|l|}
\hline Séance & $\mathbf{N}^{\mathbf{0}}$ & Loc & Intervention \\
\hline 8 & 32 & Sergueï & $\begin{array}{l}\text { aussi il a dit euh que sa maman lui avait dit que c'était dangereux à les } \\
\text { quatre ben il a dit/ ben normal/// c'est pas dangereux et::: dans le pré il:::: }\end{array}$ \\
\hline
\end{tabular}

Il tente avec une extrême maladresse de distinguer ce qui est dangereux de ce qui ne l'est pas. Mourad réussit lui à nommer l'attitude que devraient avoir les petits lapins; ceux-ci auraient intérêt à « se méfier ».

Tableau 11. «Se méfier »

\begin{tabular}{|l|l|l|l|}
\hline Séance & $\mathbf{N}^{\mathbf{o}}$ & Loc & Intervention \\
\hline 1 & 2 & Mourad & $\begin{array}{l}\text { il y avait des lapins/ ils s'appelaient Romarin/Capucine/ Neige/ Pierre/ et } \\
\text { apreur maman dit à ses lapereaux/ méfiez-vous/ je dois m'absenter pour } \\
\text { la journée }\end{array}$ \\
\hline
\end{tabular}


Théophile peut se mettre dans la situation qui est celle des lapins où le monde se partage en deux, un espace avec ennemi et un espace sans.

Tableau 12. Un point de vue « contre-factuel »

\begin{tabular}{|l|l|l|l|}
\hline Séance & $\mathbf{N}^{\mathbf{0}}$ & Loc & Intervention \\
\hline 11 & 93 & Théophile & $\begin{array}{l}\text { ben moi ce que j'aurais fait/ je serais resté carrément à la maison// je } \\
\text { serais pas sorti comme ça/ j'aurais pas d'ennemi qui pourrait me } \\
\text { manger// }\end{array}$ \\
\hline
\end{tabular}

Il ne se contente pas de raconter successivement ce que font les uns et les autres, en usant d'une simple juxtaposition (du type « lui il a fait, et lui il a pas fait »), ainsi que procèdent beaucoup d'élèves. Il recourt à l'organisation syntaxique sujet-verbe-comme-nom.

Tableau 13. Titre

\begin{tabular}{|l|l|l|l|}
\hline Séance & $\mathbf{N}^{\mathbf{0}}$ & Loc & Intervention \\
\hline 11 & 67 & Théophile & $\begin{array}{l}\text { et puis aussi moi ce que je pense de Pierre/ il est gentil mais il a } \\
\text { désobéi à sa maman// ça fait déjà deux fois dans la semaine qu'il est } \\
\text { arti alors qu'elle lui avait interdit// il aurait dû faire comme Neige } \\
\text { Capucine et Romarin/ d'aller cueillir des mûres dans le pré// }\end{array}$ \\
\hline
\end{tabular}

41 L'effort fait pour prendre du recul par rapport au simple rappel dans le désordre de tous les épisodes de l'histoire est très coûteux pour ces élèves qui ont pourtant montré qu'ils pouvaient faire des interventions dans une langue s'écartant un peu de la langue parlée familière. Leurs propos sont alors maladroits et recourent souvent à une langue familière; mais ce sont pourtant eux qui, mieux et plus que leurs camarades, arrivent à prendre des risques en parlant.

\section{Un cas particulier : la séance 3}

Nous n'affirmons pas que la situation didactique décrite ci-dessus soit la seule qui permette une telle prise de distance des élèves par rapport au simple déroulé de la course-poursuite. La séance 3 fournit un exemple intéressant d'une autre situation.

Elle comprend 433 interventions dont la très grande majorité sont extrêmement courtes (un, deux parfois trois mots). Seuls trois élèves, Malo, Marianne et Clarissa, restituent des fragments du texte source; mais ceux-ci sont très réduits et souvent éloignés les uns des autres. Un autre élève Reda arrive à se joindre à eux à la fin de la séance. Ils ont investi une situation différente de celle des autres séances, du fait du positionnement particulier du maitre : celui-ci n'y occupe pas la posture en retrait attendue. Il cadre fortement la restitution de l'histoire par de très nombreuses relances et en demande un déroulé chronologique. Ceci a pour effet de concentrer l'attention des élèves sur l'histoire et de faire passer le texte au second plan, ce qui explique le petit nombre de reprises du texte et la brièveté de ces reprises. Mais le maitre dans ses relances pose souvent des questions 
en "pourquoi ». Cette incitation conduit les élèves qui semblent avoir le plus en tête le texte à adopter une position plus distanciée par rapport au simple récit.

Marianne cite en S3-30 le texte source : « il a dit/ votre pauvre père n'est jamais revenu ». Le maitre demande ce que cela veut dire et Clarissa en S3-34 dit : « il est mort ».

En S3-74, Malo cite le propos de la maman interdisant aux lapins d'aller dans le jardin : « vous pouvez aller jouer dans le pré mais surtout n'allez pas dans le jardin de monsieur Grégory ». Il commente en S3-83 "c'est le danger ». Commence alors entre lui et Marianne un échange durant lequel ils hésitent pour savoir si Pierre a désobéi en connaissance ou non du danger : Malo « et après il savait le danger »-Marianne : « il a désobéi [...] il savait pas le danger/ alors il a désobéi » et après une relance du maitre «il a pas eu peur du danger ». Marianne à l'aide de Malo finit par résoudre la contradiction apparente entre avoir connaissance d'un danger et à se mettre en danger en désobéissant : ceux qui se mettent en danger sont ceux qui n'ont pas peur du danger.

En S3-100 le maitre demande si Pierre avait le droit d'aller dans le jardin. Pour toute réponse Malo reprend un adjectif qui figurait dans le texte source dans le syntagme «le potager interdit »; il dit en S3-102 : « interdit ».

-213 au moment où les élèves viennent d'évoquer l'épisode où Pierre manque de se faire attraper parce que son gilet s'est pris dans un filet, Clarissa fait la liaison avec l'épisode suivant : « il arrive pas à trouver le trou par où il est entré ».

Presque à la fin de la séance en se relayant l'un l'autre, mais pas toujours immédiatement, Malo, Marianne et Clarissa passent de la reprise du texte source «parce qu'ils [les chats] sont vraiment les ennemis des lapins » (Marianne, S3-253) à l'affirmation " les humains/ c'est les ennemis des lapins »(Clarissa, S3-299). Ils arrivent ainsi à généraliser à tous les hommes le comportement de monsieur Grégory et à montrer qu'ils ont compris que son attitude à l'égard de Pierre et du papa de Pierre n'est pas dictée par une animosité particulière.

Reda, un élève qui n'appartient pas au petit groupe de ceux qui reprennent des segments du texte source, parvient à prendre fugitivement de la distance avec le déroulement de l'histoire et cela relativement tardivement dans le cours de la séance. Il est intervenu 13 fois, souvent après une sollicitation directe du maitre, mais très brièvement, de manière factuelle et sans presque jamais revenir au texte source; il a signalé en S3-181 qu' « on a oublié quelque chose "; après ces prises de parole il est resté un long moment silencieux ; mais en S3-266 il met en relation deux personnages de l'histoire : " comme danger/ il y avait d'abord monsieur Grégory et après la chatte "; cette remarque est alors commentée par Clarissa (S3-267) : « s'il a la chatte et le monsieur Grégory ça va faire beaucoup pour Pierre ». Cet élève, qui n'est pas un " grand parleur » mais qui s'efforce jusqu'à la fin de participer à l'interaction verbale, même si c'est souvent de façon minimaliste, est capable au moins une fois, à l'instar de ses trois camarades de prendre du recul et de commenter la situation du héros en disant qu'elle est particulièrement problématique. La vie quotidienne a peut-être pu lui apprendre combien il est périlleux d'avoir deux adversaires à la fois! Il a réussi à prendre part à la réflexion conduite par ses trois camarades.

50 La situation de la séance 3 est donc très différente de celle des autres séances. Elle ressemble sans doute plus aux situations habituellement mises en place par les enseignants quand ceux-ci veulent vérifier si les élèves ont bien compris une histoire qui 
vient de leur être lue. Elle nous permet par comparaison de mieux comprendre la spécificité de la situation didactique qui est celle du reste du corpus.

\section{Situations didactiques favorables au développement de la compétence narrative chez des élèves ne sachant pas encore lire}

\section{Des effets de la présence en arrière-plan d'un texte écrit dans la reconstitution collective orale d'une histoire lue antérieurement}

51 Le fait d'avoir en mémoire des fragments d'une narration écrite peut à certaines conditions avoir un double effet bénéfique pour des élèves qui ne savent pourtant pas encore lire.

52 Au plan linguistique ceux-ci peuvent y puiser des expressions toutes faites; ils peuvent aussi faire passer un terme de leur lexique passif à leur lexique actif ; et ils peuvent enfin s'essayer à produire des tours syntaxiques se démarquant légèrement de ceux qui leur sont habituels.

53 Au plan cognitif tout se passe pour eux comme s'ils se trouvaient devant une tâche moins coûteuse que celle que doivent effectuer, quand ils narrent des éléments de l'histoire, ceux de leurs camarades qui n'ont pas en mémoire des «morceaux» du texte écrit. Ces derniers doivent tout à la fois retrouver des éléments de l'univers de référence en les rattachant à l'un des épisodes de l'histoire et donner certaines caractéristiques des personnages. Pour les premiers, l'univers de référence et les faits et dires des personnages sont en quelque sorte en réserve dans les fragments mémorisés.

54 Les deux phénomènes réunis permettent aux élèves qui sont dans cette situation de s'interroger sur ce que veut dire l'histoire et de prendre une distance par rapport au simple déroulé des faits narrés, tout en s'essayant à produire une langue orale moins familière. Ici certains élèves ont pu réfléchir à ce qui fonde certaines règles sociales comme l'obéissance aux parents ou jouer à se faire peur devant un monde où les grands mangent les petits, et le tout dans une langue s'écartant légèrement de l'oral dont ils usent habituellement.

\section{Caractéristiques d'une situation pouvant favoriser pour tous les élèves le développement de leur compétence narrative orale}

Nous venons d'essayer de décrire l'effet que la situation didactique dominante dans ce corpus avait pu avoir sur ceux des élèves qui avaient été capables de mémoriser des segments d'un texte écrit. Mais les élèves en question sont peu nombreux, voire même dans une ou deux classes, absents. Il s'agit d'élèves qui pour diverses raisons avaient une capacité de mémorisation supérieure à celle de leurs camarades. Il n'importe pas ici de chercher ce qui pourrait expliquer que tous les élèves ne disposent pas d'une telle capacité, mais de se demander comment la situation pourrait être modifiée afin qu'elle ne s'avère pas différenciatrice.

Il convient à la fois de garder de la situation ce qui a pu agir sur la compétence narrative de certains élèves et de la modifier pour que cette action s'exerce sur tous. La piste la plus 
évidente à suivre nous semble être d'agir pour que le maximum d'élèves puisse mémoriser des segments relativement importants du texte écrit.

On pourrait actionner deux leviers différents: l'un concerne la sélection des textes et l'autre les conditions de l'oralisation de ces textes. Le texte choisi, Danger dans le potager, était sans doute trop long et comportait trop d'épisodes. Tout en gardant ses caractéristiques linguistiques, les textes pourraient raconter l'histoire à thème constant d'un personnage fortement caractérisé aux prises avec deux ou trois évènements successifs. Après avoir été choisi, le texte (quitte à procéder sur lui à des retouches pour le simplifier) pourrait être lu, toujours sans aucun recours aux illustrations, en le segmentant et en le lisant à plusieurs reprises à plusieurs jours d'intervalle. Des courtes séances de récitation de petits passages de ce texte pourraient même être organisées, durant lesquelles seraient particulièrement sollicités les plus " petits parleurs ». Et enfin, une fois tous les élèves, y compris les plus faibles, capables de restituer presque littéralement certains segments du texte, on pourrait procéder à la restitution de l'histoire racontée par le texte en reproduisant rigoureusement à l'identique la situation didactique du corpus.

Ces étapes successives avec leurs contraintes respectives nous semblent, à la lumière de l'étude de ce corpus, susceptibles de faciliter à la fois l'appropriation par les élèves de certaines tournures linguistiques nouvelles, mais ne s'écartant que peu de celles qui leur sont habituelles, et la prise de distance par rapport à la simple narration factuelle de l'histoire. Une telle démarche pourrait être reproduite tout au long de l'année dès la moyenne section de maternelle avec plusieurs textes.

La situation didactique étudiée n'a pas été installée délibérément dans les classes. Elle est le fruit de contraintes mises en place à d'autres fins. Mais cela ne fait pas qu'elle ne puisse pas avoir des vertus heuristiques et que l'on ne puisse chercher à la reproduire avec certains aménagements, l'inscrivant ainsi dans une didactique de l'oral. Construire un enseignement raisonné de l'oral ne va pas de soi (Laparra, 2008). L'oral est évanescent et difficile de ce fait à observer et à évaluer. Des exercices systématiques du type de ce que permet l'écrit sont à cause de cela d'une conception peu aisée. L'étude d'un corpus construit à d'autres fins a permis de voir se dessiner une situation d'apprentissage pouvant être régulièrement reproduite, à condition qu'elle soit installée avec rigueur. Pour cela, il est nécessaire que les maitres aient été formés à observer comment les élèves investissent réellement les situations orales proposées par eux et qu'ils aient été sensibilisés au rôle qu'y joue la mémoire orale.

\section{BIBLIOGRAPHIE}

BAUTIER, É. (1995). Pratiques langagières, pratiques sociales. De la sociolinguistique à la sociologie du langage. Paris : L'Harmattan.

BLANCHE-BENVENISTE, C. (1997). Approches de la langue parlée en français. Gap/Paris : Ophrys. 
BONNÉRY, S. (2014). « Les livres et la manière de lire à l'école et dans la famille : réflexions à l'occasion de la parution de la liste officielle "maternelle" ». Le français aujourd'hui 185, p. 45-57. En ligne : https://www.cairn.info/revue-le-francais-aujourd-hui-2014-2-page-47.htm.

BRIAND, J. (1993). L'énumération dans le mesurage des collections. Bordeaux : Université de Bordeaux BRousseAu, G. (1998). Théorie des situations didactiques. Grenoble : Éd. La Pensée sauvage.

DUBoIS, J. (1965). Grammaire structurale du français. Le nom et le pronom. Paris : Larousse.

FRANÇOIS, D. (1974). Français parlé. Tomes 1 et 2. Paris : SELAF.

GADET, F. (1989). Le français ordinaire : Paris : A. Colin.

GROSSMANN, F. (2000) [1996]. Enfances de la lecture. Manières de faire, manières de lire à l'école maternelle. Berne : Peter Lang.

LAPARRA, M. (1982). « Sélection thématique et cohérence du discours à l'oral ». Le français moderne 50(3), p. 208-236. En ligne : https://fr.calameo.com/read/000903947b402a3b3975c? authid=fmWxzKZvzSTi.

LAPARRA, M. (2003). «Variations et usages linguistiques dans et hors de l'école ». Le français aujourd'hui 143, p. 7-16. En ligne : https://www.cairn.info/revue-le-francais-aujourd-hui-2003-4page-7.htm.

LAPARRA, M. (2008). « L'oral, un enseignement impossible? ». Pratiques 137-138, p. 117-134. En ligne : https://journals.openedition.org/pratiques/1155.

LAPARRA, M. \& MARGOLINAS, C. (2016). Les premiers apprentissages scolaires à la loupe. Louvain-laNeuve : De Boeck.

PÉROZ, P. (2010). Apprentissage du langage oral à l'école maternelle. Metz : CRDP de Lorraine.

\section{NOTES}

1. La numérotation des paragraphes est celle du texte source.

\section{RÉSUMÉS}

Comment faire pour que des élèves ne sachant pas encore lire puissent incorporer dans leurs prises de parole certaines tournures présentes dans un texte narratif leur ayant été lu à voix haute antérieurement? On étudie ici la manière dont les élèves de douze classes de grande section de maternelle restituent des éléments d'une histoire de course-poursuite dont le texte leur a été oralisé préalablement sans l'aide d'aucune image. L'analyse du corpus ainsi obtenu montre que les élèves ne s'appuient pas également sur le texte source. Ces différences s'expliquent par certaines particularités de la situation didactique dans laquelle sont placés les élèves (demande de se souvenir, exigence d'exhaustivité et non pas demande de raconter l'histoire) auxquelles s'ajoute la proximité de la syntaxe de certains énoncés du texte source avec celle de la langue parlée, proximité qui ne veut pas dire que le texte source essaye de donner une 
impression d'oral. Des pistes se dessinent pour construire des situations où le texte source peut devenir, grâce à un travail de mémorisation, suffisamment disponible pour être une ressource au moment de la prise de parole.

How can pupils still not able to read be made to insert in their speech some expressions present in a narrative text that was first read to them aloud? Here is being studied the very special way in which the pupils in twelve classes in the last year of kindergarden reproduce in turn the elements of a chase story whose text was previously to them aloud. The analysis of the corpus thus obtained reveals that not all the pupils return to the source text in the same way. Some characteristics of the situation in which the pupils are placed (they may be asked to "remember", required to be exhaustive and not asked to "tell the story"), along with the syntax of some sentences from the source text being close to that of spoken language, account for some of these differences. This provides avenues to create situations where all the pupils may try to produce oral statements slightly diverging from those they usually resort to, after being made to partially memorize the source text so as to make it more available.

\section{INDEX}

Mots-clés : mémorisation, syntaxe de l'oral, situation didactique

Keywords : memorization, oral syntax, didactic situation

\section{AUTEUR}

\section{MARCELINE LAPARRA}

Université de Lorraine, Crem, EA 3476, F-57000, France 